\title{
DFG-Schwerpunktprogramm SPP 2026 „Geometrie im Unendlichen“
}

\author{
Der Senat der Deutschen Forschungsgemeinschaft (DFG) beschloss im Frühjahr 2016 die Einrichtung eines \\ Schwerpunktprogrammes zur Erforschung unendlicher Strukturen in der Geometrie. Dieses hat im Jahr 2017 seine \\ Arbeit aufgenommen und unterstützt individuelle Forschungsprojekte, Konferenzen, Workshops und andere \\ wissenschaftliche Aktivitäten. Mittlerweile umfasst es 79 Einzelprojekte in der Differentialgeometrie, der \\ geometrischen Topologie und der globalen Analysis. Mehr als 8o Forscherinnen und Forscher an mehr als \\ 20 Universitäten in Deutschland und in der Schweiz sind an dem Programm beteiligt. \\ Ende 2020, als „Geometrie im Unendlichen“ die Hälfte seiner sechsjährigen Laufzeit erreicht hatte, gab der \\ Koordinator des Schwerpunktprogrammes, Bernhard Hanke von der Universität Augsburg, den Mitteilungen ein \\ Interview, in dem die Anfänge, die Ziele und die Organisation des Schwerpunktprogrammes beleuchtet werden. Eine \\ bearbeitete Version erscheint in dieser Ausgabe der Mitteilungen. Es folgen wissenschaftliche Beiträge von Carla \\ Cederbaum (Tübingen) und Alexander Engel (Münster), die eigene Projekte im Schwerpunktprogramm leiten und \\ ihre Forschung in allgemein verständlicher Form vorstellen. \\ Weitere Informationen über „Geometrie im Unendlichen“ sind auf der Webseite www.spp2026.de verfügbar.
}

\section{„Geometrie im Unendlichen“ erreicht wichtiges Etappenziel}

\author{
Bernhard Hanke im Gespräch mit Allyn Jackson
}

Der Titel Ihres SPPs lautet „Geometrie im Unendlichen". Was bedeutet das, und welche Arten von Geometrie gehören $\mathrm{zu}$ diesem Themenbereich?

Ein wichtiger Aspekt ist, dass wir uns nicht in erster Linie auf die klassischen Gebiete der Geometrie wie die Differentialgeometrie oder Riemann'sche Geometrie, die geometrische und die globale Analysis beziehen. Diese klassischen Bereiche untersuchen zum Beispiel geschlossene oder kompakte Mannigfaltigkeiten, relativ kleine Objekte also. In der Gruppentheorie könnte man sich mit endlichen Gruppen beschäftigen. Oder man betreibt globale Analysis auf diesen kompakten Objekten, die eine endliche Größe haben.

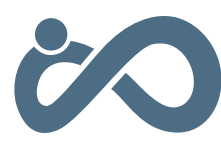

SPP 2026 GEOMETRY AT INFINITY
Wenn wir aber von endlichen Objekten zu unendlichen Objekten übergehen, treten weitere Phänomene auf. Betrachten wir etwa unendlich ausgedehnte glatte Mannigfaltigkeiten, dann müssen wir berücksichtigen, was geschieht, wenn wir uns auf der Mannigfaltigkeit ins Unendliche bewegen. Wir betrachten also Folgen von Punkten auf der Mannigfaltigkeit und untersuchen ihr Verhalten. Nähern sie sich auf sinnvolle Weise einem Punkt im Unendlichen an? Können wir das präzise fassen? Das öffnet die Tür zu vollkommen neuen Problemen und Ideen und führt auch zu der Überlegung, geometrischen Objekten ideale Ränder zuzuordnen, also Kompaktifizierungen.

Wir können auch Folgen von Mannigfaltigkeiten selbst untersuchen und fragen, ob diese gegen ein Objekt konvergieren, mit dem wir etwas anfangen können. Dann sehen wir, dass Singularitäten oder Kollapsphänomene auftreten können. In der geometrischen Topologie betrachten wir unendliche Gruppen statt endlicher Gruppen und vor allem deren Wechselwirkung mit geometrischen Eigenschaften von Objekten. Dies führt zur Untersuchung von geometrischen Objekten mit unendlichen Fundamentalgruppen. Wenn wir unendlich ausgedehnte Objekte betrachten, dann gestaltet sich die Analysis völlig anders, sodass wir auch in der globalen Analysis auf völlig andere Phänomene stoßen.

Zusammenfassend identifiziert „Geometrie im Unendlichen“ drei Themen - Konvergenz, Kompaktifizierungen und Starrheit - die in diesen klassischen Gebieten auftreten, wo man geometrische Eigenschaften untersucht. Das gibt uns ein Grundgerüst, das quer zu den klassischen Gebieten verläuft. Es ist eines der Hauptmerkmale unseres SPPs.

Können Sie von einigen Projekten erzählen, die im Rahmen von "Geometrie im Unendlichen“ gefördert werden und die Sie für besonders erfolgreich, wichtig oder auf andere Weise beachtenswert halten?

Die Anzahl der im Rahmen dieses SPPs geförderten Projekte ist zunächst einmal relativ groß. Im ersten Förder- 
zeitraum, von 2017 bis 2020, hat die DFG 33 Projekte gefördert. In der zweiten Förderperiode sind es sogar noch mehr, nämlich 46 Projekte. All die verschiedenen Themen, die ich vorhin erwähnt habe, sind bei diesen Projekten vertreten.

Eine besonders starke Forschergruppe gibt es im Bereich der geometrischen Gruppentheorie. Das ist ein aufstrebendes, sehr dynamisches Gebiet. Es gibt mehrere erfolgreiche Forschungsgruppen in Bielefeld und auch in Karlsruhe. Auf der anderen Seite unterstützen wir Arbeiten im Bereich der allgemeinen Relativitätstheorie; in der globalen Analysis studiert man geometrische partielle Differentialgleichungen, die mit der allgemeinen Relativitätstheorie in Verbindung stehen. Eine Kombination von Topologie und Geometrie, die ich sehr schön finde, ist die Erforschung und Klassifizierung von Räumen und Modulräumen geometrischer Strukturen, die man zum Beispiel auf eine fixe Mannigfaltigkeit legen kann. Dies ist eine wichtige Entwicklung, die in unserem SPP im Rahmen mehrerer Projekte gefördert wird. Hier betrachtet man nicht nur ein bestimmtes geometrisches Objekt, sondern versucht den Raum aller möglichen Strukturen zu verstehen, die ein bestimmtes geometrisches Phänomen erfassen. Man kann zum Beispiel das untersuchen, was wir Darstellungsvarietäten nennen. Eine Darstellungsvarietät besteht aus allen Darstellungen einer Gruppe im Sinne geometrischer Transformationen, die der Gruppe zugeordnet sind. In Heidelberg gibt es eine sehr starke Forschungsgruppe, die sich mit diesem Thema befasst.

Ein wichtiges Anliegen unseres SPPs ist es, Forschungsgruppen zusammenzubringen, die ansonsten womöglich keinen Kontakt hätten. Manchmal sprechen Leute aus den verschiedenen Bereichen eigentlich über denselben Gegenstand, nur in verschiedenen Sprachen. Wir haben diese Verbindungen schon im ersten Förderzeitraum recht erfolgreich durch Aktivitäten wie Workshops, Seminare und Lehrveranstaltungen unterstützt. Wenn man sich die Geschichte der Mathematik anschaut, liegt es auf der Hand, dass die Kombination von Kompetenzen aus verschiedenen Bereichen zu spektakulären Ergebnissen führen kann, die womöglich nicht erzielt würden, wenn man sich nur auf eine Denkrichtung beschränkte.

Wie wird ein SPP ins Leben gerufen, und wie verläuft es, wenn die Finanzierung gesichert ist?

Unter den verschiedenen koordinierten Fördermaßnahmen der DFG ist das SPP die älteste. Es unterscheidet sich von anderen Förderungen wie Sonderforschungsbereichen oder Graduiertenkollegs. Um ein SPP zu beantragen, verfasst eine Gruppe von Wissenschaftlerinnen und Wissenschaftlern zunächst eine sogenannte Initiative zur Einrichtung des Programms. In unserem Fall besteht die Gruppe aus Christian Bär aus Potsdam, Anna Wienhard aus Heidelberg, Burkhard Wilking aus Münster und mir. Wir haben 2015 beschlossen, eine SPP-Initiative zu schreiben. Wir legten die genannten Forschungsschwerpunkte fest - Konvergenz, Kompaktifizierungen und Starrheit und erklärten, warum wir glauben, dass ein Schwerpunktprogramm zur Weiterentwicklung dieser Bereiche beitra- gen würde. Und wir skizzierten, was unserer Ansicht nach in den sechs Förderjahren geschehen könnte.

Das ist vollkommen anders als bei der Beantragung eines Sonderforschungsbereichs, wo man schon ganz am Anfang konkrete Forschungsanträge stellen muss. Das SPP hat ein zweistufiges Verfahren, das ich sehr effizient finde. Wir legten den Rahmen fest, die DFG reservierte ein bestimmtes Budget dafür, und dann lag es an den einzelnen Forscherinnen und Forschern, innerhalb dieses Rahmens Anträge zu stellen.

Initiativen für SPPs können auf allen von der DFG geförderten Gebieten vorgeschlagen werden, und der Senat der DFG entscheidet dann, welche Initiativen unterstützt werden. Sobald die DFG die Einrichtung eines SPPs beschließt, hält sie für die ersten drei Jahre einen bestimmten Geldbetrag bereit. Danach wird entschieden, ob die Förderung für weitere drei Jahre fortgesetzt wird, wobei diese Entscheidung eher automatisch erfolgt.

Das ist eine fantastische Ausgangslage, denn wir haben die Sicherheit, dass uns innerhalb dieses Forschungsgebietes ein bestimmtes Budget zur Verfügung steht, mit dem wir unsere Ziele realisieren können. Außerdem fließt die SPP-Förderung unabhängig vom üblichen DFG-Budget für Mathematik, sodass unser SPP nicht die Mittel für andere Bereiche der Mathematik schmälert.

Nachdem unser SPP eingerichtet worden war, verschickte die DFG eine Aufforderung zur Einreichung von Anträgen mit einer Beschreibung des Themas „Geometrie im Unendlichen“. Das Format für Anträge ist sehr ähnlich wie bei den DFG-Anträgen für Einzelprojekte im Normalverfahren, mit zwei Unterschieden. Zum einen bitten die Antragstellenden darum, dass ihr Antrag im Rahmen des SPP berücksichtigt wird, und erklären, warum er zum Thema des SPP passt. Zum anderen gibt es eine Frist für die Einreichung von Anträgen für das SPP, während es für die Einreichung von Anträgen für Einzelprojekte bei der DFG keine Frist gibt.

Nach Ablauf der Frist schickte die DFG alle Anträge an eine internationale Gruppe von Gutachterinnen und Gutachtern. Wir wussten, wer zu dieser Gruppe gehörte; es war nicht geheim. Nach drei oder vier Monaten hielten wir ein Evaluationskolloquium ab, bei dem jeder, der einen Antrag eingereicht hatte, ihn den anderen Antragstellenden und dem Gutachtergremium vorstellen konnte.

Bei diesem Evaluationskolloquium müssen eine Menge Leute gewesen sein.

Ja, auf dem Kolloquium zur ersten Förderperiode, für die wir 6o-70 Anträge bekommen hatten, waren 80-9o Leute. Es fand im Dezember 2016 in Augsburg statt.

Nach der Vorstellung ihrer Anträge verließen die Antragstellenden das Kolloquium. Dann trafen sich die Gutachterinnen und Gutachter, um die Anträge zu bewerten und der DFG Vorschläge zu machen, welche gefördert werden sollten. Wir hatten ein hochkarätiges internationales Gutachtergremium, und sein Urteil war entscheidend für eine hohe Qualität der geförderten Projekte und eine gute Mischung der Forschungsgebiete. Bei diesem Treffen war 


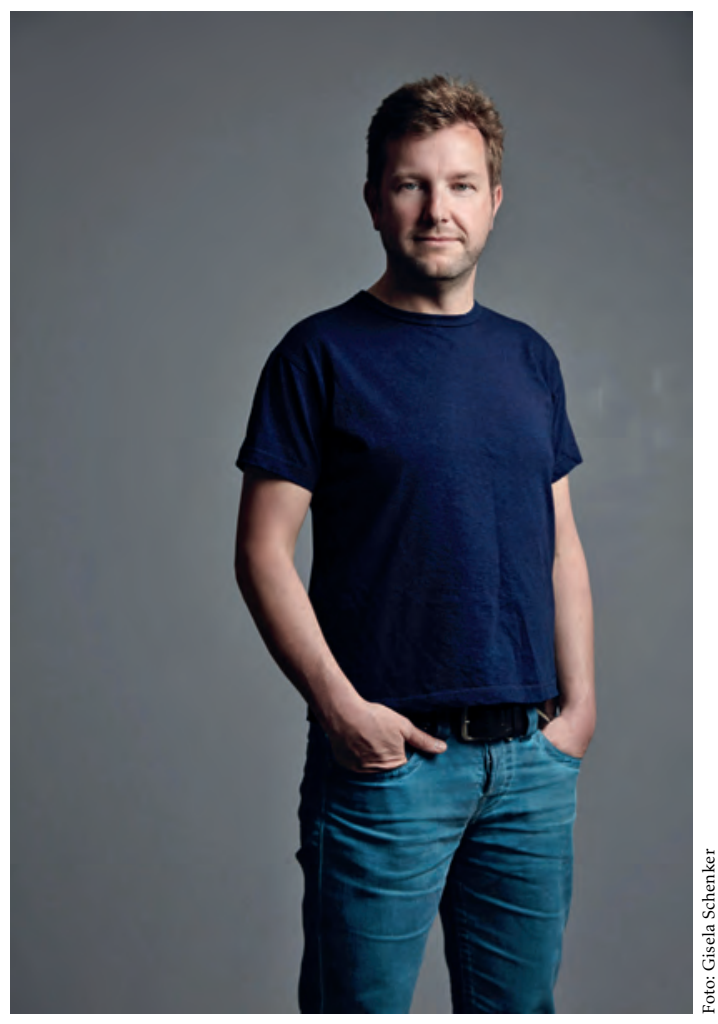

Bernhard Hanke

auch ein Vertreter der DFG anwesend, der kalkulieren konnte, wie die Anträge am besten in das Budget passten.

Das Evaluationskolloquium für die zweite Förderperiode war für März 2020 geplant, musste aber wegen der Pandemie abgesagt werden. Das Gutachtergremium traf sich stattdessen zu einer virtuellen Sitzung, in der es die Anträge diskutierte und die Vorschläge für die DFG erarbeitete.

Jeder, dessen Projekt gefördert wird, ist Mitglied im SPP. Zusätzlich zu den Einzelprojekten gibt es das Koordinatorprojekt, für das nur ich als Koordinator des Programms mich bewerben konnte. Das Koordinatorprojekt enthält Mittel, die nicht nur für mich, sondern für alle Mitglieder von "Geometrie im Unendlichen“ gedacht sind. Die anderen drei Mitglieder des Programmkomitees - Bär, Wienhard und Wilking - und ich haben auf unserer Website eine Rubrik eingerichtet, in der SPP-Mitglieder Mittel aus dem Koordinatorprojekt beantragen können. Sie könnten zum Beispiel eine einwöchige Konferenz mit fünfzig Teilnehmenden, von denen die Hälfte aus dem Ausland kommt, vorschlagen und einen bestimmten Betrag für Reisen und Hotels geltend machen. Und wir können das aus dem Koordinatorprojekt recht großzügig unterstützen. In der ersten Förderperiode konnten wir alle derartigen Vorschläge finanzieren, und es gab zahlreiche Aktivitäten, wie Sie auf unserer Website sehen können.

Eine größere Veranstaltung war unsere SPP-Konferenz, die ebenfalls aus dem Koordinatorbudget gefördert wurde. Diese fand mit Plenarvorträgen von gut zehn international renommierten Expertinnen und Experten im Frühjahr 2019 in Münster statt. Jedes SPP-Forschungsprojekt war bei dieser Konferenz vertreten. Eine weitere wichtige Veranstaltung, die zu Beginn jeder Förderperiode stattfindet, ist das sogenannte Kickoff-Meeting. Wir hatten am Anfang des ersten Förderzeitraums ein Kickoff-Meeting in Potsdam, bei dem sich jedes Projekt vorgestellt hat, damit sich alle kennenlernen konnten. Leider mussten wir wegen der Pandemie das Kickoff-Meeting für die zweite Förderphase verschieben. Wir planen es für den Herbst 2021. Im Idealfall würden wir es gerne als Präsenzveranstaltung durchführen, denn gerade die Kontakte und das Netzwerk sind das Besondere an diesem SPP und sorgen dafür, dass es mehr ist als nur die Summe der Einzelprojekte. Und diese Kontakte und das Netzwerk werden durch das Koordinatorprojekt zusammengehalten.

Der Wegfall der persönlichen Kontakte ist für jeden schwierig, aber ganz besonders für ein Programm wie das SPP, denn die Kontakte sind das, was man eigentlich fördern will.

Ja. Wir stehen nun schon seit etwa zehn Monaten vor diesem Problem. Natürlich haben die SPP-Mitglieder einige Zoom-Meetings anstelle von persönlichen Treffen abgehalten, aber die enorme Aktivität, die während der ersten Förderperiode stattfand, ist im März oder April 2020 wirklich zum Erliegen gekommen. Die Leute warten einfach auf bessere Zeiten. Ich hoffe wirklich sehr, dass wir in absehbarer Zeit zu diesem Niveau von Aktivitäten zurückkehren können.

Bei einer Zoom-Konferenz können die Leute über ihre Forschung sprechen, und das ist auch gut so. Bei einer Präsenzveranstaltung dagegen sind es die Dinge, die zwischen den Vorträgen passieren - auf dem Flur, in den Kaffeepausen oder beim Abendessen -, durch die die Veranstaltung tatsächlich mehr bietet, als ,anderen Leuten von der eigenen Forschung zu erzählen“. Für Gruppen aus verschiedenen Richtungen der Mathematik ist es viel einfacher, den ersten Kontakt herzustellen und herauszufinden, dass sie eigentlich gemeinsame Forschungsinteressen haben und nur eine andere Sprache benutzen, wenn die Leute physisch zusammenkommen und sich bewegen und unterhalten können. Dies ermöglicht ungeplante Begegnungen, die bei Zoom-Meetings naturgemäß nicht vorkommen.

Natürlich sind wir in gewisser Weise in einer luxuriösen Lage. Wir können Zoom-Treffen abhalten und weiterarbeiten. Es gibt Teile der Gesellschaft, die sehr viel stärker von der Pandemie betroffen sind als die Wissenschaft.

Positiv ist, dass wir uns viel stärker an die Nutzung von Zoom gewöhnt haben als vor der Pandemie, und das eröffnet Möglichkeiten für den Austausch in einem größeren Rahmen. Heutzutage gibt es viele Seminare, die regulär über Zoom stattfinden und Menschen zusammenbringen, die auf verschiedenen Kontinenten arbeiten. Dadurch ergeben sich neue Möglichkeiten für die wissenschaftliche Zusammenarbeit. Eine Kombination aus persönlichen Treffen und Online-Forschungsseminaren ist vielleicht sogar besser als das, was wir vor der Pandemie hatten. Jede Krise eröffnet Chancen für neue Entwicklungen. 
Eines der Ziele des SPP ist es, den Bedürfnissen des wissenschaftlichen Nachwuchses gerecht zu werden. Wie funktioniert das bei „Geometrie im Unendlichen“?

In einem Einzelantrag für das SPP können Mittel für Promovierende und Postdocs beantragt werden, und diese Förderung des wissenschaftlichen Nachwuchses spielt eine sehr wichtige Rolle. Außerdem ermutigen wir bei Konferenzen und Workshops dazu, Organisationskomitees zu bilden, die jüngere Wissenschaftlerinnen und Wissenschaftler mit erfahreneren zusammenbringen. Wir haben schon viele Konferenzen veranstaltet, die von einer Gruppe von Forschenden in verschiedenen Karrierestufen organisiert wurden.

Eine weitere Besonderheit des SPP ist, dass Nachwuchswissenschaftlerinnen und -wissenschaftler eigene Stellen beantragen können, die es ihnen ermöglichen, die Leitung eines eigenen Forschungsprojekts zu übernehmen. Ihr Name ist mit dem Projekt verknüpft, und ihr Gehalt wird von der DFG bezahlt. Sie können die eigene Stelle an jede gewünschte Universität mitnehmen. Das hat sich als eine sehr gute Möglichkeit erwiesen, die Karriere junger Menschen zu fördern. Einige von ihnen haben auch den nächsten Schritt gemacht und noch während unserer ersten Förderperiode eine feste Stelle angetreten.

Das kann für junge Menschen eine schwierige Übergangsphase sein.

Ganz genau. Der Übergang von einer befristeten Stelle zu einer unbefristeten oder Tenure-Track-Stelle ist tatsächlich die kritische Phase. An dieser Stelle ist das SPP für jüngere Wissenschaftlerinnen und Wissenschaftler eine große Hilfe. Wenn man eine Professur hat, dann verfügt man über ein eigenes Budget, da ist es vielleicht weniger wichtig, DFG-Mittel zu haben.

Das SPP bietet jungen Leuten fantastische Möglichkeiten. Wenn sie zum Beispiel ein Gebiet entdecken, über das sie gerne mehr wissen würden, können sie ein Lernseminar vorschlagen, damit sie sich vernetzen und neue wissenschaftliche Kontakte knüpfen können. Dafür können sie beim Koordinatorprojekt Unterstützung beantragen. Und das läuft eher informell ab, weil die Anträge auf Förderung durch das Koordinatorprojekt an das Programmkomitee - Bär, Wienhard, Wilking und mich - gestellt werden und nicht über die DFG laufen müssen.

Für junge Menschen ist die Mitgliedschaft in einem SPP eine großartige Möglichkeit, ihre wissenschaftliche Karriere voranzutreiben. Tatsächlich war das auch bei mir der Fall. Als ich Postdoc in München war, hatte ich zusammen mit Thomas Schick ein Forschungsprojekt, das in einem SPP namens "Globale Differentialgeometrie“ angesiedelt war, das von 2003 bis 2010 bestand. Ich habe erst später gemerkt, wie wertvoll das für meine Karriere war.

Die SPPs müssen Maßnahmen zur Gleichstellung der Geschlechter berücksichtigen. Wie ist das in Ihrem SPP umgesetzt?
Wir haben in unserem Koordinatorbudget einen bestimmten Betrag, der für Gleichstellungsmaßnahmen vorgesehen ist. Wenn also zum Beispiel eine Forscherin ihr Kind zu einer Konferenz mitbringen möchte, können wir für die Kinderbetreuung oder sogar für eine Begleitperson aufkommen. Eine solche Unterstützung haben wir schon einige Male geleistet. Außerdem haben wir in Heidelberg eine Konferenz mit dem Titel „Women in Geometry“ veranstaltet, die vor allem auf den Aufbau von Netzwerken und Kontakten unter Wissenschaftlerinnen zielte. In der zweiten Förderperiode werden wir eine neue Maßnahme starten, ein Mentoring-Programm für Wissenschaftlerinnen. Es soll jüngere Forscherinnen mit älteren zusammenbringen, entweder durch regelmäßige persönliche Treffen oder per Zoom, um die jüngeren Kolleginnen zu ermutigen, in der Wissenschaft zu bleiben. Auf diese Weise Vorbilder zu geben kann sehr wichtig sein.

Sie sind sehr begeistert von dem SPP-Fördermodell in der Mathematik. Aber vielleicht gibt es auch Änderungen, die Ihrer Meinung nach an dem SPP-Modell vorgenommen werden sollten, damit es der Mathematik noch besser gerecht würde.

Der größte Teil der Fördermittel im SPP wird für die Vergütung von Promovierenden und Postdocs verwendet, aber das sind alles befristete Stellen. Und das ist das größte Problem bei solchen Förderprogrammen, dass es keine langfristige Perspektive gibt - nicht nur in der Mathematik, sondern auch in anderen Wissenschaften. Wir erhöhen die Chancen von jüngeren Leuten, eine feste Stelle zu bekommen, aber die Zahl der Festanstellungen wird durch das SPP nicht erhöht. Wir haben zwei Förderperioden von jeweils drei Jahren, was sehr schön ist und uns vieles erreichen lässt. Aber am Ende der sechs Jahre haben wir wissenschaftliche Spitzenkräfte, die eine Festanstellung suchen. Man sollte solche Forschungs- und Fördermaßnahmen durch bessere Perspektiven für Festanstellungen ergänzen.

Es gibt einige Schritte in diese Richtung, zum Beispiel die Schaffung von mehr Tenure-Track-Stellen oder Juniorprofessuren, auf die man sich in einem früheren Karrierestadium bewerben kann. Leider erhöht sich die Gesamtzahl der unbefristeten Stellen in der Mathematik dadurch nicht, zumindest nicht in nennenswertem Umfang. Der Zuwachs reicht bei Weitem nicht, um die Spitzenforscherinnen und -forscher aufzufangen, die aus einem sechsjährigen Förderprogramm wie unserem SPP hervorgehen.

Die Universitäten werden von den Ländern finanziert, die DFG hauptsächlich vom Bund. Eine Einrichtung wie die DFG kann keine Professorinnen und Professoren einstellen oder unbefristete Stellen vergeben. Die Erhöhung der Zahl der Festanstellungen ist also ein schwieriges politisches Problem.

Glauben Sie, dass Deutschland von etwas wie dem CNRS [Centre National de la Recherche Scientifique] in Frankreich profitieren würde?

Definitiv. CNRS-Stellen sind unbefristet und ermöglichen es den Leuten, ihre Forschung voranzutreiben, nur ohne 


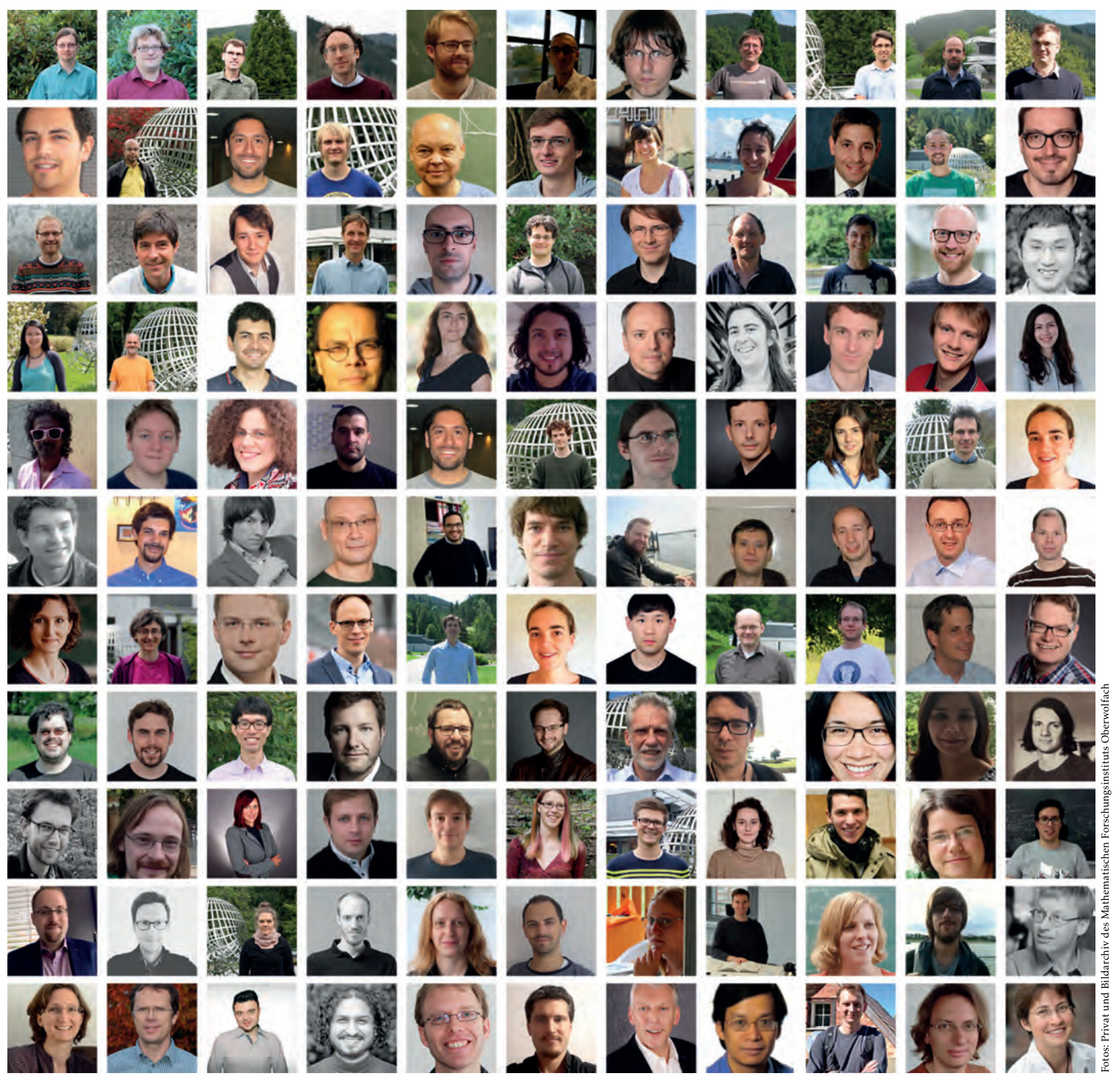

Mitglieder des SPP2O26

all die Pflichten wie Lehre und Verwaltung, die Professuren mit sich bringen. Die Gesellschaft investiert Gelder in die Ausbildung des wissenschaftlichen Nachwuchses, und ich halte es für sinnvoll, ihm die Möglichkeit zu geben, in der Forschung zu bleiben, damit die Gesellschaft auch von ihrer Investition profitiert. Einige wenige junge Spitzenkräfte aus unserem SPP bekommen Festanstellungen, aber es gibt viele andere, die Deutschland verlassen, um Stellen in anderen Ländern anzutreten. Das ist aus globaler Perspektive natürlich auch in Ordnung, aber es wäre gut, ihnen die Möglichkeit zu geben, in Deutschland zu bleiben und ihre Forschung fortzusetzen.
Das bringt mich auf einen anderen Aspekt. Das SPP trägt auch zur internationalen Sichtbarkeit der Geometrieforschung in Deutschland bei. Es handelt sich zwar um ein nationales Förderprogramm, aber es gibt Möglichkeiten, Verbindungen zwischen internationalen Forscherinnen und Forschern aufzubauen, und eines unserer SPPProjekte ist tatsächlich in der Schweiz angesiedelt. Über das internationale Gutachtergremium - das Gremium, das die SPP-Anträge begutachtet - werden Forschende außerhalb Deutschlands auf die hier stattfindende Forschung aufmerksam. Wir fördern auch internationale Workshops und Konferenzen, die im Ausland stattfinden. Zum Bei- 
spiel waren wir 2019 Mitsponsor eines großen Treffens zur Differentialgeometrie bei MATRIX, einer Forschungsstation in Australien, in der Nähe von Melbourne. Solche Aktivitäten können von unserem Koordinatorprojekt unterstützt werden. In dieser Hinsicht ist es sehr flexibel.

Wie ist es für Sie, das SPP zu leiten? Gibt es viel Verwaltungsarbeit, viel Bürokratie?

Mit dem SPP konnte ich eine Teilzeitassistentin einstellen, Alexandra Linde, und sie hilft mir sehr bei der Verwaltung. Dazu gehört die Organisation der Evaluationskolloquien und die Abwicklung der Kostenerstattung an Personen, die Koordinatormittel für Konferenzen verwenden. All das wäre ohne die wertvolle Hilfe von Alexandra nicht zu schaffen.

Ein weiterer Teil der Verwaltungsarbeit, der eher zur wissenschaftlichen Seite gehört und ziemlich spannend war, war das Einrichten der SPP-Website. Das ist wirklich ein wesentlicher Teil der Infrastruktur. Für mich war es das erste Mal, dass ich so etwas gemacht habe.

Es klingt vielleicht ein bisschen seltsam, aber ich genieße die Arbeit. Es ist wie eine große Party. Ich bin in Kontakt mit Forschungsgruppen gekommen, die ich vorher nicht so wahrgenommen habe, und mir ist klar ge- worden, dass sie wirklich gute Arbeit leisten. Mein Blick auf die Forschung in Deutschland und auf die Geometrie im Allgemeinen hat sich sehr erweitert. Das begrüße ich sehr.

Wir vom Programmkomitee sind diejenigen, die das Geld verteilen. Wir machen also die Leute glücklich! Auf die konkrete Forschung haben wir keinen Einfluss, denn die diesbezüglichen Entscheidungen werden innerhalb der einzelnen Forschungsprojekte getroffen. Wir haben einige der Highlights bei den Veranstaltungen organisiert, zum Beispiel die SPP-Konferenz, und das war fantastisch, weil wir wussten, dass unser Budget es uns erlaubt, eine Konferenz auf höchstem Niveau zu organisieren.

Dinge wie das Einrichten der Webseite, das Organisieren des Kickoff-Meetings oder auch dieses Interview, das wir gerade führen, liegen außerhalb der üblichen wissenschaftlichen Arbeit, und das macht mir Spaß. Für mich ist Wissenschaft mehr, als sich nur auf das eigene Forschungsprojekt zu konzentrieren. Wir haben eine gewisse Verantwortung, anderen zu vermitteln, was wir tun, und das spielt auch im SPP eine Rolle. Ich persönlich mag das sehr. Die Arbeit im SPP ist eine perfekte Kombination von Verwaltungsarbeit und wissenschaftlichen Ergebnissen, ein ideales Zusammenspiel von beidem.

Bernhard Hanke (Promotion 1999) studierte Mathematik und theoretische Physik an der LMU München, der University of Cambridge und der Universität Bonn. Längere Forschungsaufenthalte verbrachte er an der University of Notre Dame, an der Universität Göttingen, an der TU Berlin, am IMPA (Rio de Janeiro), am IHES (Bures-sur-Yvette) sowie am Courant Institute of Mathematical Sciences (New York). Nach einer Professur an der TU München ist er seit 2010 Inhaber des Lehrstuhls für Differentialgeometrie an der Universität Augsburg.

\title{
Geometrisch definierte asymptotische Koordinaten in der Allgemeinen Relativitätstheorie
}

\author{
Carla Cederbaum
}

\begin{abstract}
Gemeinsam mit Jan Metzger (Universität Potsdam) leite ich im Rahmen des SPP „Geometrie im Unendlichen“ ein Projekt an der Grenze zwischen Geometrischer Analysis und Mathematischer Relativitätstheorie. In diesem Projekt beschäftigen wir uns mit der Geometrie des asymptotischen Bereichs von „relativistischen Anfangsdaten“, also wortwörtlich mit deren Geometrie im Unendlichen. Genauer befassen wir uns mit verschiedenen Blätterungen des asymptotischen Bereichs und mit dazugehörigen Erhaltungsgrößen sowie deren Koordinaten(un-)abhängigkeit.
\end{abstract}

(Relativistische) Anfangsdaten $(M, g, K)$ bestehen aus einer Riemannschen Mannigfaltigkeit $(M, g)$, hier dreidimensional, sowie einem symmetrischen $(0,2)$-Tensorfeld $K$ auf $M$. Wie der Name nahelegt, beschreiben solche Anfangsdaten den „Anfangszustand“ $M=\{t=0\}$ einer Raumzeit, also ei- ner zeit-orientierten Lorentzschen Mannigfaltigkeit; $g$ ist dann die von der Raumzeitmetrik induzierte Riemannsche Metrik, das Tensorfeld $K$ ist die zweite Fundamentalform (oder „extrinsische Krümmung“) der betrachteten Hyperfläche $M=\{t=0\}$ bezüglich deren zukunftsgerichteter Nor- 